\title{
El manejo editorial de los manuscritos recibidos en la Revista Médica de Chile
}

T.T.T.: Things Take Time («Todo Toma Tiempo») The editorial handling of manuscripts
submitted to Revista M édica de Chile

This Editorial describes the steps followed by a manuscript when it is submitted to Revista Médica de Chile: its reception, format checking as requested in Instructions to Authors, and the editors' decision whether it is considered suitable for the purposes of this journal or not; the selection of peer reviewers, a direct contact with them to ask for their willingness to review this particular manuscript; an analysis by the editors of the reviewers' criticisms leading them to the decision of whether to accept it in the current version, or to return it to the authors with a request to prepare a new corrected version, or a definitive rejection; the editors' review of a corrected version (that may require again the opinion of the external reviewers) and the final decision to accept it or not; printing of the manuscript, two successive galley proofs reviewed by authors and editors; and the final printing of the journal with its simultaneous reproduction in the web page www.scielo.cl. Roughly $70 \%$ of the manuscripts are returned to the authors offering them the opportunity to resubmit a corrected version, $12 \%$ are definitively rejected and $20 \%$ are accepted in their first version. The mean time taken for an accepted manuscript since its first submission until it appears printed is currently 7.6 months. Having only part time editors and a time-limited secretarial staff, efforts to shorten this time are difficult to implement, although electronic mail and fax are increasingly being used in this editorial process (Rev Med Chile 2004: 132: 7-10).

(Key Words: Journalism, medical; Manuscripts, medical; Peer review; Periodicals)

$\mathrm{C}$ uando los autores entregan un manuscrito a una revista médica, aspiran legítimamente a que sea publicado. Una vez que lo entregaron, tienen que esperar la decisión de los editores. Durante ese lapso -que siempre parece demasiado largo- es natural que sientan curiosidad por saber qué está ocurriendo con su manuscrito. Su inquietud se acentúa cuando necesitan demostrar productividad en publicaciones científicas. En ciertas épocas del año aumentan las consultas sobre el estado de los manuscritos, coincidiendo con las fechas en que las universidades califican a sus académicos o los investigadores deben rendir informes a organismos que financiaron su investigación. Algunas revistas satisfacen esta demanda proporcionando en su página web datos sobre la etapa del proceso en que se encuentran los manuscritos. Pero ese sistema exige recursos administrativos con que cuentan muy pocas revistas.

El manejo de los manuscritos en la Revista Médica de Chile se ajusta a las recomendaciones internacionales sobre cómo seleccionarlos y cuál es el formato en que deberían publicarse. Sus 
editores tienen la responsabilidad de revisar los documentos que reciben, seleccionar los que consideran atingentes a los propósitos de la revista, decidir en cuál Sección se incluirán, programar su fecha de publicación y revisar las pruebas de impresión. Para cumplir esta responsabilidad los editores necesitan formarse un juicio técnico sobre cada manuscrito que revisan. Esto se consigue a lo largo de un proceso ordenado y es importante que los autores de manuscritos y los lectores de la Revista conozcan sus etapas:

I Etapa: Recepción del manuscrito, revisión del cumplimiento de requisitos formales, decisión sobre su atingencia a la revista y asignación de revisores externos.

El manuscrito es recibido por la secretaria de la Revista, quien revisa si cumple requisitos formales explícitos en las instrucciones a los autores: número de copias, calidad de las figuras, duplicado en diskette, firmas de los autores en los documentos de Guía de Exigencias y Responsabilidad de Autoría. Si detecta alguna omisión, lo advierte a los autores y cuando ha sido corregida coloca el manuscrito en un sobre especial y lo inscribe en una base de datos. A partir de ese momento, el manuscrito queda clasificado como documento confidencial, accesible sólo para la secretaria y los editores. Esta recepción» (que no contiene juicios sobre el contenido del manuscrito) es certificada por escrito a los autores.

Los editores hacen una primera revisión del manuscrito para decidir si el tema tratado y su presentación son adecuados para la Revista. En caso afirmativo lo clasifican como candidato a una determinada Sección. Para evaluar la calidad del contenido solicitan la opinión de expertos ( «evisión externa por pares»), contando con una base de datos que considera su especialización, el lugar y grupo de trabajo, la calidad, oportunidad y cronología de sus revisiones previas. Desde enero de 2002 todos los artículos de investigación son enviados a dos o tres revisores y los de otra naturaleza (casos clínicos, revisiones de temas, artículos de posición o especiales, etc.) son enviados a un revisor externo. Antes de esa fecha, los artículos de investigación se enviaban generalmente a un solo revisor externo y muy pocos de los demás tipos de trabajos tenían revisión externa.

La secretaria de la Revista consulta telefónicamente o por correo electrónico a los revisores elegidos si están dispuestos a recibir ese manuscrito. Este contacto previo, personalizado, está en práctica desde 1997 y ha mejorado la receptividad» de los revisores. Cuando un revisor declina recibir un manuscrito, los editores eligen otra alternativa y la secretaria repite el proceso. Antes de 1997 no se solicitaba previamente su anuencia sino que se enviaban los manuscritos con la respectiva carta/solicitud. Como consecuencia, algunos manuscritos llegaban a los revisores en momentos inoportunos, incluso durante ausencias en el extranjero, causando retrasos involuntarios en sus informes.

A los revisores externos se les envía una copia del manuscrito, las instrucciones de cómo esperan los editores que planteen su opinión y el formulario en que deben expresarla. Estos documentos se entregan confidencialmente, por correo electrónico (cuando lo aceptan los revisores), o mediante mensajero (para revisores en Santiago), o por vía postal (fuera de Santiago). Cuando el revisor ha sobrepasado el plazo asignado, la secretaria lo contacta para saber si será posible contar con su informe. En caso negativo y si los editores deciden que es importante contar con la opinión de un nuevo revisor externo, es necesario repetir el proceso descrito.

II Etapa: Recepción de los informes de los revisores externos, análisis por los editores y toma de decisiones.

Una vez recibidos los informes, los editores los estudian cotejándolos con el manuscrito, para formarse un juicio de fondo. Esto debe conducirlos a: a) aceptar la versión que enviaron los autores y decidir su publicación; b) devolver el manuscrito a sus autores, solicitándoles que preparen una nueva versión en la cual solucionen las observaciones de los revisores externos; o c) rechazar definitivamente su publicación. En las alternativas a) y b) los editores agregan sus propias observaciones y piden cambios en la presentación formal, para adaptarlo a las normas y el estilo de la Revista. 
III Etapa: Revisión de la nueva versión del manuscrito.

Los autores deciden si aceptan las observaciones planteadas a su manuscrito o prefieren destinarlo a otra revista. La mayoría de las revistas médicas aceptan hasta tres meses como plazo máximo para considerar una versión corregida como un manuscrito ya analizado, en que se requiere revisar las modificaciones hechas. En cambio, si se sobrepasa ese plazo, el documento se considera como un manuscrito nuevo y se le somete a todas las etapas ya descritas para su versión inicial.

Los editores deciden si la nueva versión es aceptable. Antes, pueden solicitar a uno o más de los revisores externos que opinen sobre la pertinencia de los cambios hechos. Si persisten críticas relevantes, los editores insisten a los autores que las corrijan, devolviéndoles su manuscrito por segunda vez.

Una vez aceptado el manuscrito, ello se comunica al autor que hace de contacto con la Revista y se programa su mes de publicación. En el momento en que esto ocurre, ya están en prensa uno o dos números futuros de la Revista, por lo cual el manuscrito no tendrá cabida antes de dos meses más. Durante ese lapso, es sometido a una revisión final por los editores, quienes hacen las indicaciones que guiarán al impresor de la Revista para diseñar su formato. Simultáneamente, se envía una copia al editor encargado de los resúmenes en inglés, quien traduce o corrige el resumen propuesto por los autores. Desde enero de 2002 se pone a disposición de los autores la nueva versión del resumen en inglés, solicitándoles su aprobación o propuesta de cambios.

\section{Etapa: Impresión de la Revista.}

La versión definitiva del manuscrito es enviada a los impresores de la Revista, quienes controlan su reproducción en una imprenta. En esta etapa es importante la correcta presentación de la copia en diskette, porque reduce el riesgo de errores en la transcripción del texto.

Una primera prueba de impresión es puesta a disposición de los autores, a quienes se solicita su revisión con énfasis en los datos numéricos, las tablas, la ubicación de las figuras y sus leyendas.
Hasta diciembre de 2001 las pruebas de imprenta eran accesibles sólo para los autores de Santiago, mientras que actualmente se emplean el fax y el correo electrónico para facilitar su llegada rápida a los autores de otras ciudades, en el país y el extranjero. A todos se les exige hacer sus correcciones en un plazo no mayor de tres días, porque después de ellos la prueba de imprenta es revisada por uno de los editores, y vuelve a los impresores.

Una segunda prueba de impresión es revisada solamente por los editores, quienes verifican las correcciones hechas y autorizan la impresión definitiva. En el mes siguiente, los suscriptores de la Revista Médica de Chile reciben el producto final de este proceso, impresa «en papel»y la pueden encontrar también en versión electrónica en el sitio web http://www.scielo.cl.

La evolución del proceso editorial en la Revista Médica de Chile ha causado una mayor carga administrativa. Ello ha sido inevitable porque para conseguir una respuesta oportuna de los revisores externos es conveniente un contacto directo y personalizado, y se requiere una mayor participación de los autores en el perfeccionamiento de sus manuscritos, tanto antes de aceptarlos como durante su impresión. El recargo de trabajo se ha acentuado aún más al recibirse un número creciente de manuscritos. En el primer semestre del año 2003 aumentó en 30\% el número de manuscritos recibidos, en comparación con el semestre equivalente del año 2000 (148 vs 114, respectivamente). La proporción de manuscritos rechazados (12\%) no tuvo variación, pero se duplicó la proporción de manuscritos que fueron devueltos a los autores para que hagan modificaciones importantes. El número de manuscritos publicados fue similar en ambos períodos, porque está limitado por la cantidad de páginas (promedio 120) que puede tener cada número de la Revista.

A pesar del mayor número de participantes en el proceso (particularmente revisores) y del aumento del número de manuscritos recibidos, el tiempo que han demorado los manuscritos aceptados, desde que se recibieron por primera vez hasta que fueron publicados, no ha variado: 7,5 meses (rango 4 a 13) en 2000 y 7,6 meses (rango 3 a 12) en 2003. Sería difícil implementar otras medidas de perfec- 
cionamiento editorial con la dotación actual de personal, particularmente de secretaría. En muchas gestiones administrativas se emplean el fax y el correo electrónico, pero hay revisores y autores que todavía prefieren relacionarse con la Revista mediante mensajero o por correo postal.

\section{Corolario}

Los editores, autores, revisores, el personal administrativo de la Revista y sus impresores, influimos en mayor o menor medida en el tiempo que demora un manuscrito, desde ser recibido hasta verse publicado. Queremos que este lapso se acorte, pero un proceso con las características descritas no puede acelerarse ilimitadamente, sin arriesgar que se perjudique su calidad.

Piet Hein (1905-1996) fue un científico danés que destacó como matemático (autor de la ssuper elipsoide»), como inventor (contribuyó a la concepción y difusión del «diseño escandinavo»en mueblería) y como escritor. En uno de sus poemas pareció retratar el mensaje de esta Editorial:

\section{«T.T.T.}

Put up in a place where it's easy to see the cryptic admonishment T.T.T.

When you feel how depressingly slowly you climb,

it's well to remember that Things Take Time»

Humberto Reyes B, Editor Joaquín Palma $H$, Max Andresen $H$, Editores Asociados 